\title{
Real-Time Monitoring of Nicotine Release Behavior from Smokeless Tobacco (Snus) Based on Fiber Optic Sensing Technology
}

\author{
Peng $\mathrm{Li}^{1}$, Shitong Zeng ${ }^{1}$, Jianxun Zhang ${ }^{1}$, Yi Shen ${ }^{2}$, Shihao Sun ${ }^{1 *}$, Yongli Zong ${ }^{1}$, Jianping Xie ${ }^{1}$, \\ Dingzhong Wang ${ }^{1}$, and Jing Yang ${ }^{2}$ \\ ${ }^{1}$ Zhengzhou Tobacco Research Institute of China National Tobacco Corporation, Zhengzhou, China. \\ 25hanghai New Tobacco Product Research Institute, Shanghai, China. \\ e-mail: sunsh@ztri.com.cn
}

\begin{abstract}
Introduction: To strengthen the safety control of smokeless tobacco (snus) products, an accurate method to test the nicotine release behavior from snus is very necessary. Methods: A laboratory-made flow-through device was designed to simulate snus using condition in the mouth, which was integrated with a commercial drug dissolution analyzer. Nicotine was gradually released from snus and dropped into the release solution. The release solution was analyzed by ultraviolet spectroscopy, and the spectral signal input to the system was detected by fiber optic probe and automatically processed by computer. The nicotine release behavior diagram was thus obtained. Also, the nicotine dissolution rate coefficient $(\alpha)$ could be calculated. Results: The results show that there was a great linear relationship between the absorbance and concentration of a nicotine standard solution at $260 \mathrm{~nm}$ in the range of $10-80 \mu \mathrm{g} / \mathrm{mL}$. The relative standard deviation (RSD) of nicotine release at several time periods was between $10.35 \%$ and $12.74 \%$, indicating good repeatability. Approximately $17-85 \%$ of nicotine was released from 16 commercial snus products. The $\alpha$ values were between 0.008 and 0.071 . Conclusions: This nicotine dissolution analyzer was designed by integrating fiber optic sensing technology, chemical spectroscopic analytical technology, and computer data processing technology. The sampling time and measurement time intervals of this method had a precision of several seconds. Thus, the design objective of the fiber optic sensing nicotine dissolution analyzer was achieved; that is, in vitro, continuous, real-time, online analysis of nicotine dissolution behavior of snus.
\end{abstract}

KEYWORDS: Snus, nicotine dissolution behavior, fiber optic sensing technology, dissolution

\section{INTRODUCTION}

nus is a subcategory of oral smokeless tobacco products, which are processed into fine particles. The products are usually placed between upper lip and gum and do not require expectoration during use. Products are available both loose and in individually portioned sachets. Tobacco components are dissolved in saliva, released into the human oral cavity, and absorbed through the oral mucosa (1). In recent years, and with the continuous strictness of the world's tobacco control policy, snus has become an important supplement to tobacco products in North America and Northern Europe (2). The analysis methods of chemical composition and formulation of snus have also become a hot topic in tobacco research in recent years (3-4). The main alkaloid contained in tobacco is nicotine. The dissolution rate and dissolution amount of nicotine are important indicators of safety control of snus products. The experiments on the nicotine release behavior in vitro can be used to assess the risk of snus and to develop methods for quality control.

Because the monitoring of nicotine dissolution from snus is of great significance, the study on the dissolution behavior of nicotine is one of the main focuses on snus research. In the 1990s, it was reported that the dissolution behavior of nicotine could be analyzed using a self-made dialysis device or a self-made supported liquid membrane device combined with an ultraviolet (UV) spectrophotometer (5-6). The two methods above are relatively simple, and the dissolution rate of nicotine was

*Corresponding author 
greatly affected by the dialysis bag or the supported liquid membrane, resulting in large measurement fluctuations. Therefore, it was difficult to accurately reflect nicotine dissolution.

The drug dissolution test is a method to measure the dissolution rate and degree of solid pharmaceutical preparations such as tablets, capsules, and granules under certain conditions. It is an instrumental method to simulate the disintegration and dissolution in the stomach and intestines, which is used to evaluate the bioequivalence and bioavailability of drugs. Moreover, it is also an important indicator for evaluating drug quality before clinical use.

In 2013, Li et al. (7) developed an in vitro releasing device capable of simulating nicotine release from snus into oral cavity, and an in vitro method was developed to analyze the nicotine release behavior from snus by this laboratorymade device. This device could distinguish the difference in nicotine release among different samples; however, the device had no program control functions. Therefore, it was cumbersome to operate and difficult to apply practically.

The drug dissolution analyzer is, as its name implies, an apparatus to test drug dissolution. Development of the drug dissolution analyzer generally goes through three stages. The first stage is dominated by manual sampling combined with a UV spectrophotometer or the use of high performance liquid chromatography (HPLC). The second stage is composed of a peristaltic pump in the drug dissolution apparatus combined with an online automatic analysis; however, there have been tubing adsorption problems, washing difficulties, and data lag issues, which greatly affect practical application of the devices. The third stage contains the fiber optic drug dissolution analyzer, which integrates fiber optic technology, spectroscopy technology, computer technology, remote sensing technology, and drug analysis technology, forming an optical-mechanical-electrical integrated fiber optic drug dissolution analyzer. Thus, the in situ real-time automatic processing analysis was achieved. Since the 1990s, many labs have focused on the development of such fiber optic drug dissolution analyzers (8). In 2010, a new generation of China's "Fiber Optic Sensing Drug Dissolution Analyzer" was successfully developed and put into use in Chinese Pharmacopoeia (9).

Therefore, we aimed to design an in vitro dissolution device capable of simulating the oral dissolution of nicotine from snus according to the oral consumption characteristics of snus. We combined the device with the drug dissolution analyzer to analyze the dissolution behavior of nicotine from snus to provide references for the detection and regulation of nicotine dissolution behavior in snus products.

\section{MATERIALS AND METHODS}

\section{Materials, Reagents, and Instruments}

Sixteen commercially available snus products in North Europe and North America were studied. Table 1 shows samples information in details. All samples were sealed and frozen $\left(-18^{\circ} \mathrm{C}\right)$ before testing.

Table 1. Weight, Water Level, pH and Nicotine Content of 16 Snus Samples

\begin{tabular}{|c|c|c|c|c|c|c|}
\hline No. & $\begin{array}{c}\text { Weight } \\
(\mathbf{g})\end{array}$ & $\begin{array}{c}\text { Water } \\
(\%)\end{array}$ & $\mathrm{pH}$ & $\begin{array}{c}\text { Nicotine } \\
(\mathrm{mg} / \mathrm{g}, \text { wet } \\
\text { weight) }\end{array}$ & $\begin{array}{c}\text { Cumulative } \\
\text { release } \\
(\%)\end{array}$ & $\alpha$ \\
\hline 1 & 0.9077 & 49.19 & 7.41 & 9.03 & 50 & 0.031 \\
\hline 2 & 0.8142 & 14.33 & 6.34 & 14.26 & 74 & 0.048 \\
\hline 3 & 1.0547 & 26.07 & 5.50 & 21.47 & 54 & 0.052 \\
\hline 4 & 0.8115 & 42.51 & 7.59 & 23.77 & 52 & 0.033 \\
\hline 5 & 0.4907 & 11.61 & 6.03 & 16.87 & 43 & 0.015 \\
\hline 6 & 0.8988 & 20.45 & 5.86 & 14.18 & 47 & 0.024 \\
\hline 7 & 0.4692 & 45.42 & 5.83 & 19.06 & 44 & 0.024 \\
\hline 8 & 0.7818 & 8.49 & 7.41 & 17.72 & 85 & 0.071 \\
\hline 9 & 0.7172 & 30.18 & 6.62 & 16.50 & 63 & 0.052 \\
\hline 10 & 0.5996 & 29.09 & 7.35 & 9.32 & 53 & 0.027 \\
\hline 11 & 0.6028 & 15.42 & 6.71 & 17.32 & 65 & 0.048 \\
\hline 12 & 0.8654 & 45.66 & 6.40 & 11.36 & 64 & 0.044 \\
\hline 13 & 0.2816 & 9.73 & 7.31 & 18.12 & 82 & 0.067 \\
\hline 14 & 0.8014 & 39.15 & 7.79 & 18.24 & 60 & 0.032 \\
\hline 15 & 0.2879 & 6.33 & 8.43 & 16.68 & 17 & 0.008 \\
\hline 16 & 0.7154 & 41.91 & 7.88 & 15.74 & 68 & 0.057 \\
\hline
\end{tabular}

Nicotine (purity $\geq 99.0 \%$, National Tobacco Quality Supervision and Inspection Center of China National Tobacco Corporation, Zhengzhou, Henan, China), triethylamine (chromatographic purity, Chemicals North West Technologies GmbH, Duesseldorf, Germany), disodium hydrogen phosphate (purity $\geq 99.0 \%$, Tianjin Zhiyuan Chemical Reagent Co., Ltd., Tianjin, China), potassium dihydrogen phosphate (purity $\geq 99.5 \%$, Tianjin Kaitong Chemical Reagent Co., Ltd.), purified water, and methanol (chromatographically pure, Shanghai High Purity Reagent Co., Ltd. Shanghai, China).

Aqueous needle filters $(13 \times 0.22 \mu \mathrm{m}$, Shanghai Anpel Scientific Instrument Co., Ltd.), brown chromatographic bottles $(2 \mathrm{~mL}$, Shanghai Anpel Scientific Instrument 
Co., Ltd.), and an analytical balance (CP2245, $0.0001 \mathrm{~g}$, Sartorius, Göttingen, Germany) were used in this study.

The instruments used in this study included a laboratorymade fiber optic nicotine dissolution analyzer modified on a commercial drug dissolution analyzer (FODT-101, Shanghai Focus Analytical Instruments Co., Ltd.), an HPLC device (Waters 2695-2996, Waters, Milford, MA, USA), an ultra-pure water generator (Milli-Q A10, Millipore Co., Burlington, MA, USA), and an ultrasonic cleaner (8894, Cole Parmer, Vernon Hills, IL, USA).

\section{Structure of Fiber Optic Nicotine Dissolution Analyzer}

The laboratory-made fiber optic nicotine dissolution analyzer was modified based on the fiber optic drug dissolution analyzer. A laboratory-made flow-through device was designed to simulate snus using condition in mouth, which was integrated with the commercial drug dissolution analyzer. Nicotine was gradually dissolved and released from snus and dropped into the collection cup. The released solution in cup was analyzed by ultraviolet spectroscopy. Also, the spectral signal input to the system was transferred by fiber optic probe and automatically processed by computer. The nicotine dissolution behavior diagram was thus obtained. A diagram of the laboratorymade fiber optic nicotine dissolution analyzer is shown in Figure 1.

\section{Working Principle of Fiber Optic Nicotine Dissolution Analyzer}

The dissolution solution of snus is a multi-component mixed system. Based on the principle of absorbance addition, the following equation was obtained (not considering matrix effects):

$$
A_{\lambda}=\sum_{i=1}^{n} \varepsilon_{\lambda}^{i} C_{i} l
$$

where $A_{\lambda}$ is the total absorbance value of soaking solution at one wavelength, $\varepsilon$ is the molar attenuation coefficient, $C$ is the concentration, and $l$ is the optical length.

We define $\triangle A$ as the absorbance of impurities in the soaking solution as follows: $\Delta A=A^{\text {Soaking }}-A^{\text {Nic }}$, where, $A^{\text {Soaking }}$ is the total absorbance of the soaking solution, and $A^{\text {Nic }}$ is the absorbane of nicotine. We assume that $k$ $=\left(\Delta A_{1}-\Delta A_{3}\right) /\left(\Delta A_{2}-\Delta A_{3}\right)$, where $\Delta A_{1}$ is the absorbance of impurities at $\lambda_{1}$, chosen as the maximum absorption wavelength of nicotine; $\Delta A_{2}$ is the absorbance of impurities at $\lambda_{2}$, where nicotine has no absorption and impurities have a strong absorbance; and $\Delta A_{3}$ is the absorbance of impurities at $\lambda_{3}$, where nicotine has no absorbance and impurities have no absorbance or a weak absorbance. Therefore, we can get the equation: $A_{1}{ }^{\text {Nic }}=A_{1}^{\text {Soaking }}-k \Delta A_{2}+(k-1) \Delta A_{3}$.

Because we choose $\lambda_{2}$ as nicotine no absorbance and impurities strong absorbance and $\lambda_{3}$ as nicotine no absorbance and impurities no absorbance or weak absorbance, that is to say $\Delta A_{2}$ is approximately equal to $A_{2}{ }^{\text {Soaking }}$ and $\Delta A_{3}$ is approximately equal to $A_{3}{ }^{\text {Soaking }}$. So, we can finally get the following equation: $A_{1}$ Nic $=A_{1}$ soaking $-k A_{2}^{\text {Soaking }}+(k-1) A_{3}^{\text {Soaking }}$.

In summary, the real-time concentration of nicotine can be calculated from the total absorbance values at $\lambda_{1}, \lambda_{2}$, and $\lambda_{3}$, and the nicotine concentration curve (nicotine dissolution curve) over time can be plotted using the computer operating software.

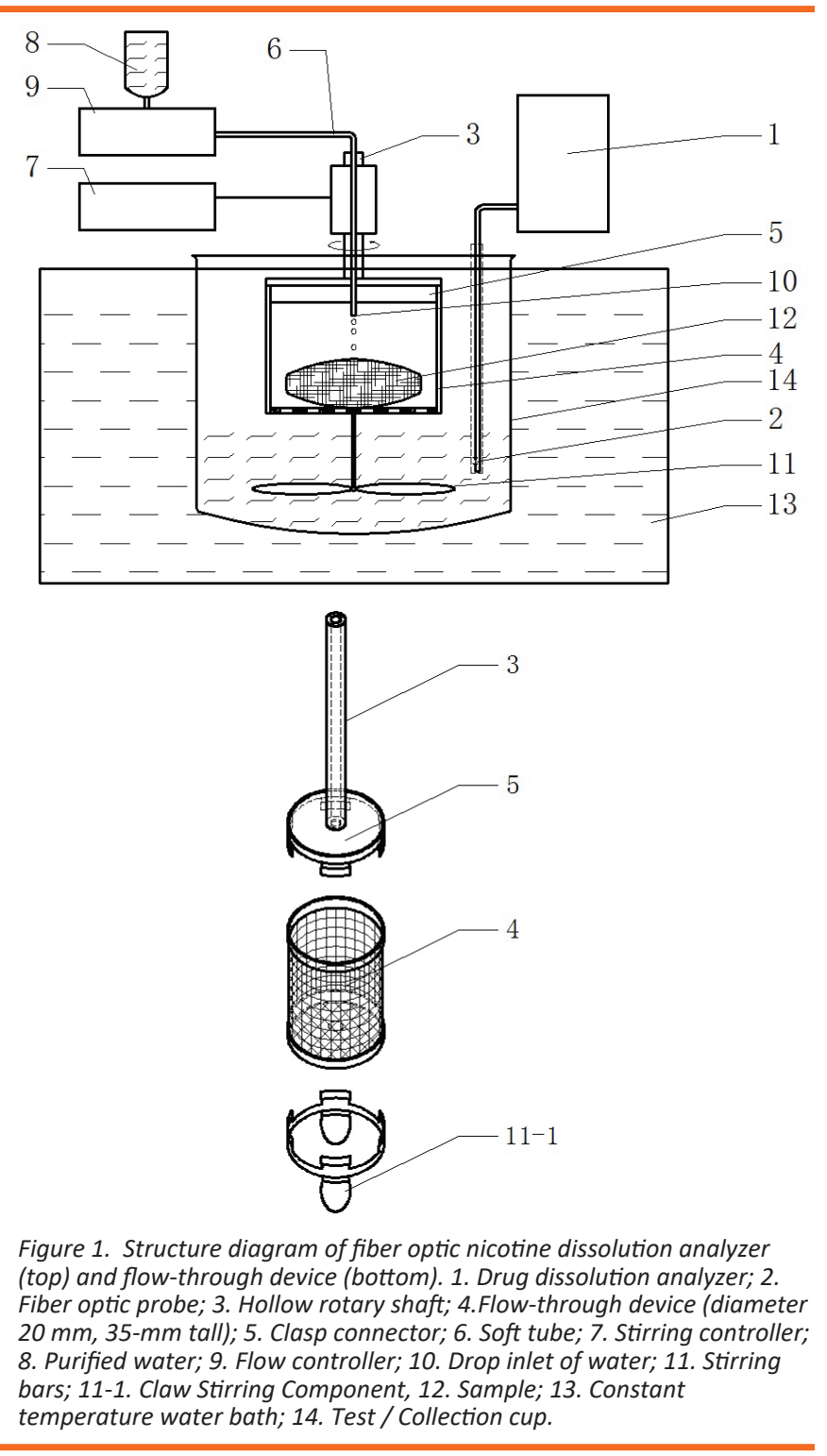




\section{Preparation of Solutions}

\section{The preparation of liquid phase for HPLC}

The potassium dihydrogen phosphate $(3.388 \mathrm{~g})$ and disodium hydrogen phosphate (3.533 g) were weighed accurately and dissolved in pure water. Triethylamine (2 $\mathrm{mL})$ and methanol $(220 \mathrm{~mL}$ ) were then added, after which the solution was diluted to $1 \mathrm{~L}$ with purified water. The solution was filtered through a $0.45 \mu \mathrm{m}$ filter prior to use.

\section{Preparation of nicotine standard solution}

A series of nicotine standard solutions were prepared according to the principle that the linear range of concentration should be close to and greater than the concentrations of all samples. The standard nicotine amount $(0.5 \mathrm{~g})$ was dissolved in pure water and diluted to $100 \mathrm{~mL}$ with purified water. Then, samples of 2, 4, 8, 12, and $16 \mathrm{~mL}$ were taken and diluted to $1000 \mathrm{~mL}$, forming nicotine standard solutions with concentrations of 10 , $20,40,60$, and $80 \mu \mathrm{g} / \mathrm{mL}$, respective. All solutions were protected from light at $4{ }^{\circ} \mathrm{C}$.

\section{Experimental Procedures}

The experimental procedures were as follows:

a. Weigh sample and put it into $150 \mathrm{~mL}$ water, soak at $37{ }^{\circ} \mathrm{C}$ with stirring speed $100 \mathrm{rpm}$. After $1 \mathrm{~h}$, remove sample and obtain the soaking solution, then measure the absorbance at $\lambda_{1}, \lambda_{2}$, and $\lambda_{3}$.

b. Transfer the soaking solution to a chromatography bottle and measure the nicotine concentration using HPLC and record nicotine concentration.

c. Make a nicotine standard solution of the same concentration, and measure the absorbance at $\lambda_{1}, \lambda_{2}$, and $\lambda_{3}$. Calculate $k$ and enter the value into the software for alkaloid analyzer (Zhengzhou, trial version, Henan, China).

d. Turn on the instrument, set water bath to $37{ }^{\circ} \mathrm{C}$, open the release test module, add $150 \mathrm{~mL}$ water to the dissolution cup, then immerse the probe into water (degassed by ultrasonic for $1 \mathrm{~h}$ before using). Weigh another sample and place it in the dissolution cell, which is above water.

e. Click start, add purified water drops to the sample at a fixed flow rate as $0.2 \mathrm{~mL} / \mathrm{min}$, and stir at the same time. The instrument starts measuring and recording every 10 seconds, and the results are shown in the software's window in real time.

f. After $1 \mathrm{~h}$, stop the experiment and save the files. g. According experimental data (corrected for changing media volume), calculate accurate nicotine release percentage and release rate coefficient $\alpha$, which are also shown in the software's window.

The accurate nicotine release percentage was calculated as (nicotine concentration in $\mu \mathrm{g} / \mathrm{mL} \times$ the volume of release solution in $\mathrm{mL}$ ) $\div$ (nicotine level in $\mathrm{mg} / \mathrm{g}$, determined by Coresta recommended method \#87 $\times$ sample weight in $\mathrm{g}) \times 100(10)$.

The release rate coefficient $\alpha$ is calculated by the Weibull model: $Y=1-\exp (-\alpha t) \mu$, where $Y$ is the cumulative dissolution fraction, $\alpha$ is the scale parameter that determines the time scale of the dissolution process, and $\mu$ is the shape parameter that determines the shape of the curve. The curve is exponential when $\mu=1$, is S-shaped when $\mu>1$, and is an exponential curve with a steep initial slope when $\mu<1$. In this study, the value of $\mu$ was 1 . The shape of the fitting curve mainly depended on $\alpha$, meaning that the cumulative dissolution rates of nicotine over time were determined by $\alpha$. The value of $\alpha$ reflected the speed of nicotine dissolution. Therefore, $\alpha$ was defined as the nicotine dissolution rate coefficient, which was used to evaluate the dissolution rates of nicotine.

\section{RESULTS AND DISCUSSION}

\section{Determination of Reference Wavelength}

The wavelengths were selected based on the following principles: $\lambda_{1}$ is the maximum absorption wavelength of nicotine; nicotine has no absorption at the wavelength of $\lambda_{2}$ and impurities have strong absorption; there is no absorption for nicotine at the wavelength of $\lambda_{3}$, and there is no absorption or a weak absorption for impurities. Based on the above principle, $\lambda_{1}, \lambda_{2}$, and $\lambda_{3}$ were chosen as 260,290 , and $550 \mathrm{~nm}$, respectively. The full wavelength spectrum of the soaking solution is shown in Figure 2. The full wavelength spectrum of the nicotine standard solution with same concentration is shown in Figure 3.

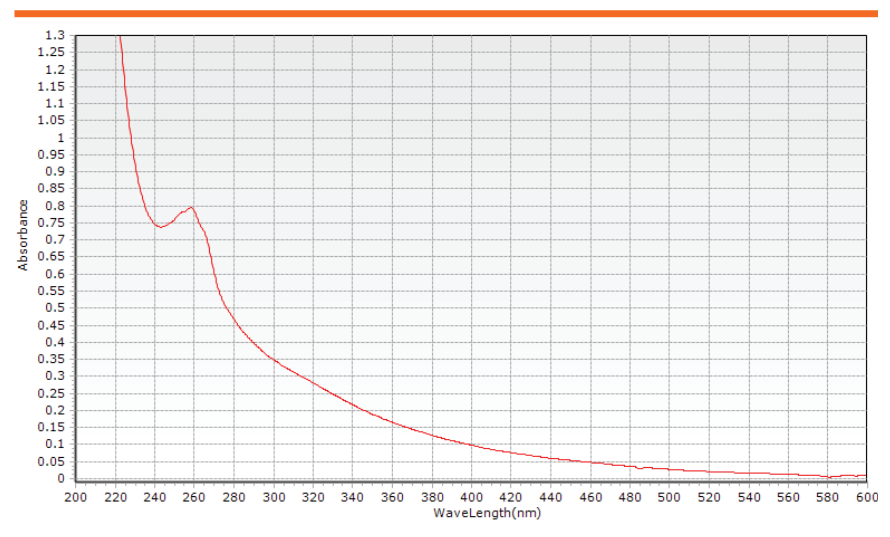

Figure 2. Full wavelength spectrum of the soaking solution. 


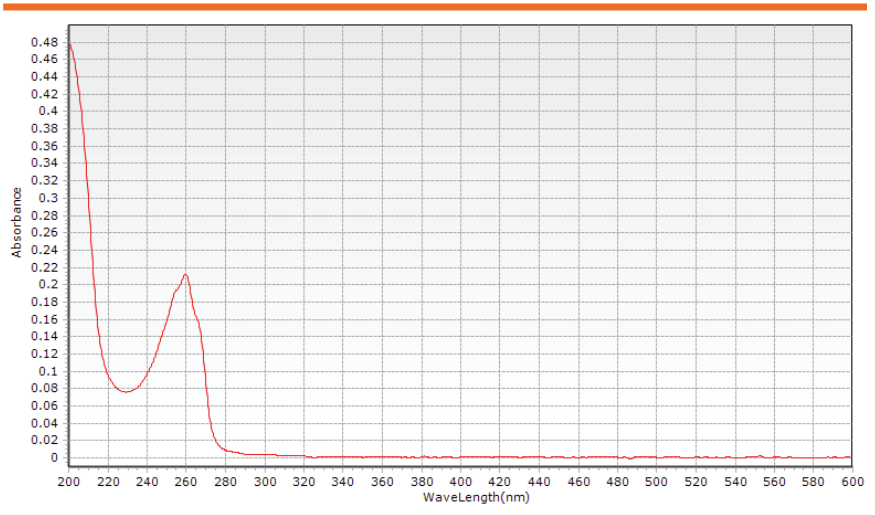

Figure 3. Full wavelength spectrum of the nicotine standard solution with same concentration.

\section{The Stability, Linearity, and Range of Absorbance}

The relationship between the absorbance and concentration of the nicotine standard solution was analyzed using the fiber optic nicotine dissolution analyzer with different concentrations $(10-80 \mu \mathrm{g} / \mathrm{mL})$ at three $\mathrm{pH}$ levels $(5.29,6.98$, and 8.67). The calibration curves, linear equations, and correlation coefficients were automatically displayed and shown in the software's window. Results are shown in Figure 4, which shows that the method is suitable for solutions with different $\mathrm{pH}$ ranges. Absorbance values for all nicotine standard solutions were stable at $260 \mathrm{~nm}$. At this wavelength and concentration range, there was a good linear relationship between the absorbance and concentration of the nicotine standard solution.

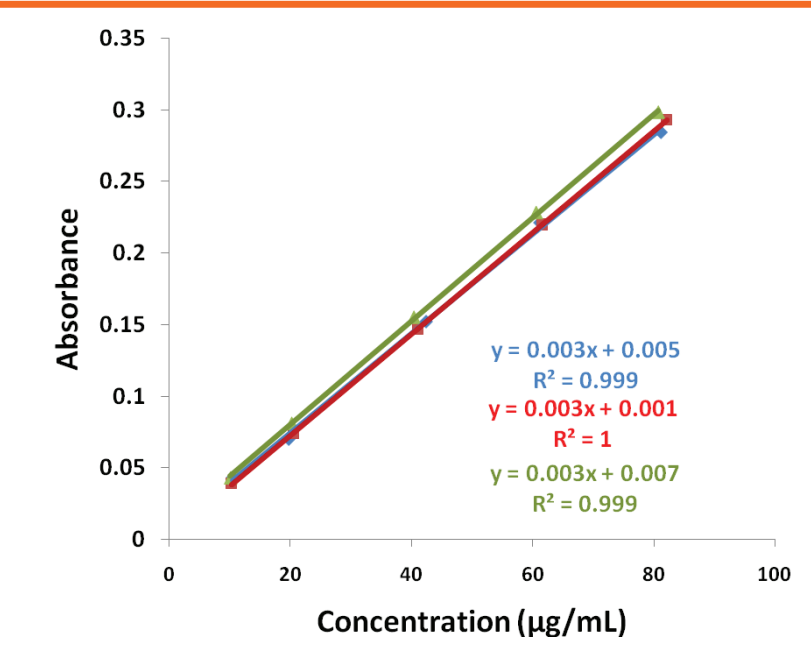

Figure 4. Relationship between absorbance and concentration of nicotine standard solution at 3 different values ( $p H 5.29$ in blue, $\mathrm{pH} 6.98$ in red, $\mathrm{pH}$ 8.67 in green).

\section{Repeatability}

The samples to be tested were thawed at room temperature for 2 hours. The average mass of the snus samples was calculated based on 100 pouches of snus products. The samples with masses in the range of the average mass ( $\pm 1 \%$ ) were used for future experiments. These samples were tested for dissolution of nicotine according to the experimental procedures. Parallel experiments were performed 5 times. The relative standard deviation (RSD) of the cumulative release rate of nicotine at $10,20,30,40,50$, and 60 mins was calculated separately. RSDs of nicotine release at each time period were $11.75 \%, 12.74 \%, 11.71 \%, 11.69 \%, 11.34 \%$, and $10.35 \%$, indicating a good repeatability of the method.

\section{Actual Sample Detection}

The samples to be tested were thawed at room temperature for 2 hours. The average mass of the snus samples was calculated based on 100 pouches of snus products. The samples whose masses were in the range of the average mass $\pm 1 \%$ were used for future experiments. Qualified samples were used for nicotine dissolution test in vitro according to the experimental procedures. Parallel experiments were performed three times, based on which the $k$ values were calculated. The experimental results of the in vitro nicotine dissolution test are shown in Figure 5.

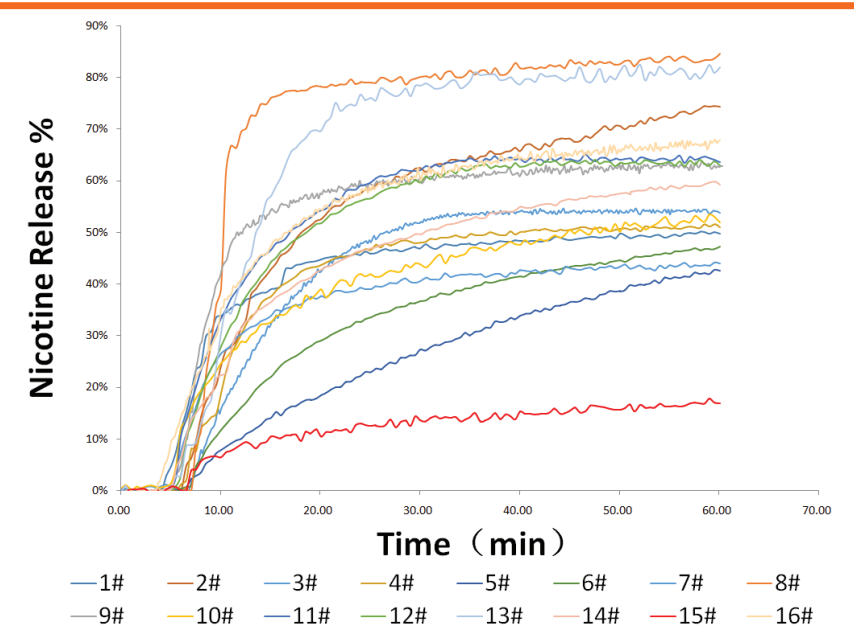

Figure 5. Nicotine release behaviors of 16 snus products (1\#-16\#) studied.

The results on the dissolution behaviour of nicotine from the four foreign commercial snus samples were all fitted using the Weibull model. The goodness of fit $\left(R^{2}\right.$ value) for all samples were greater than 0.96 , indicating a good fit based on the Weibull model. Therefore, the Weibull model can be used to evaluate and predict the dissolution rate of nicotine from snus at various times. The experimental results are shown in Figure 4.

It can be seen from Figure 4 that the degree of nicotine dissolution for all of the samples was between $17 \%$ and $85 \%$. The minimum nicotine dissolution rate coefficient was 0.008 , and the maximum was 0.071 , a 
huge difference of approximately nine times, indicating that the degree and rate of dissolution of nicotine from different manufacturers and brands are quite different. Therefore, the in vitro nicotine dissolution test can be quickly and effectively conducted using the method in this study, which is of great methodological significance to studying the influencing factors and the degree of nicotine dissolution from snus.

\section{CONCLUSIONS}

An in vitro method for analyzing nicotine dissolution behavior from snus was established based on fiber optic sensing technology. A nicotine dissolution analyzer was designed by integrating fiber optic sensing technology, chemical spectroscopic analytical technology, and computer data processing technology. A flow-through device was designed to simulate snus using conditions in mouth, which was integrated with a commercial drug dissolution analyzer. There was a linear relationship between the absorbance and concentration of a nicotine standard solution at $260 \mathrm{~nm}$ in the range of 10-80 $\mu \mathrm{g} / \mathrm{mL}$. The RSD values of nicotine release at several different time periods were between $10.35 \%$ and $12.74 \%$, indicating a good repeatability of the method. The method was applied to 16 commercial snus products. The results showed that approximately $17-85 \%$ of nicotine was released from samples, with $\alpha$ values between 0.008 and 0.071 .

\section{FUNDING}

This research was supported by the China National Tobacco Corporation (10201501004).

\section{DECLARATION OF INTERESTS}

The authors disclosed no conflicts of interest related to this article.

\section{REFERENCES}

1. Connolly, G. N.; Alpert, H. R. Trends in the use of cigarettes and other tobacco products, 2000-2007. JAMA. 2008, 299, 26292630. DOI: 10.1001/jama.299.22.2629.

2. Digard, H.; Errington, G.; Richter, A.; McAdam, K. Patterns and behaviors of snus consumption in Sweden. Nicotine Tob. Res. 2009, 11, 1175-1181. DOI: 10.1093/ntr/ntp118.

3. Zhang, J.; Li, P.; Zong, Y.; Sun, S.; Wang, S.; Xie, J. Analysis of total nicotine, free nicotine and tobacco-specific N-nitrosamines in foreign oral smokeless tobacco products. Tob. Sci. \& Tech. 2011, 291, 44-48. DOI: 10.3969/j.issn.1002-0861.2011.10.011.

4. Digard, H.; Gale, N.; Errington, G.; Peters, N.; McAdam, K. Multianalyte approach for determining the extraction of tobacco constituents from pouched snus by consumers during ues. Chem. Centr. J. 2013, 15, 255-261. DOI: 10.1186/1752-153x-755.

5. Nasr, M. M.; Reepmeyer, J. C.; Tang, Y. B. In vitro study of nicotine release from smokeless tobacco. Aoac Int.1998, 81, 540-543.

6. Luque-Pérez, E.; Ríos, A.; Valcárcel, M.; Danielsson, L. G.; Ingman, F. Analysis of solid samples using supported liquid membranes:a method for the evaluation of the release of nicotine from Swedish snuff. Anal. Chim. Acta. 1999, 387, 155164. DOI: 10.1016/s0003-2670(99)00077-x.

7. Li, P.; Zhang, J.; Sun, S.; Xie, J.; Zong, Y. A novel model mouth system for evaluation of in vitro release of nicotine from moist snuff. Chem. Centr. J. 2013, 7, 176-184. DOI: 10.1186/1752-153x7-176.

8. Nir, I.; Lu, X. In Situ UV fiber optics for dissolution testing - what, why, and where we are after 30 years. Dissolution Technol. 2018, 3, 70-77. DOI: 10.14227/DT250318P70.

9. State Pharmacopoeia Commission. Pharmacopoeia of the People's Republic of China. Volume 4. Pharmacopoeia Science and Technology Press: Beijing, 2015, p 931.

10. Scientific Committee of Cooperation Centre for Scientific Research Relative to Tobacco (Coresta). Determination of nicotine in tobacco products by GC-MS, Coresta Recommended Method No. 87, Cooperation Centre for Scientific Research Relative to Tobacco, Paris, France, 2018. 